CERN-TH/2001-038

\title{
Towards An Accurate Calculation of the Neutralino Relic Density
}

\author{
Takeshi Nihei ${ }^{1}$, Leszek Roszkowski ${ }^{1,2}$ \\ and Roberto Ruiz de Austri ${ }^{1}$ \\ ${ }^{1}$ Department of Physics, Lancaster University, Lancaster LA1 4YB, England \\ ${ }^{2}$ TH Division, CERN, CH-1211 Geneva 23, Switzerland
}

\begin{abstract}
We compute the neutralino relic density in the minimal supersymmetric standard model by using exact expressions for the neutralino annihilation cross section into all tree-level final states, including all contributions and interference terms. We find that several final states may give comparable contributions to the relic density, which illustrates the importance of performing a complete calculation. We compare the exact results with those of the usual expansion method and demonstrate a sizeable discrepancy (of more than 10\%) over a significant range of the neutralino mass of up to several tens of $\mathrm{GeV}$ which is caused by the presence of resonances and new finalstate thresholds. We perform several related checks and comparisons. In particular, we find that the often employed approximate iterative procedure of computing the neutralino freeze-out temperature gives generally very accurate results, except when the expansion method is used near resonances and thresholds.
\end{abstract}

CERN-TH/2001-038

February 2001 


\section{Introduction}

One of the most exciting unresolved issues in particle physics and cosmology is the nature of dark matter (DM) in the Universe. Cosmological and astronomical observations have put, over the years, significant restrictions on its expected properties. In particular, it is widely expected that a hypothetical candidate should be in the form of cold DM (CDM), while a combination of several measurements has narrowed the range of its relic abundance to

$$
0.1 \lesssim \Omega_{\mathrm{CDM}} h^{2} \lesssim 0.3
$$

where $\Omega_{\mathrm{CDM}}$ is the ratio of the CDM relic density to the critical density and $h \approx 0.7$ is a parameter in the Hubble constant $H_{0}=100 \mathrm{~h} \mathrm{~km} / \mathrm{sec} / \mathrm{Mpc}$ [1]. Constraints from the Big Bang Nucleosynthesis [2] and, recently, from observations of the cosmic microwave background radiation [3, 4] have strongly restricted the abundance of baryonic DM; for example, Ref. [5] quotes the range $\Omega_{b} h^{2}=0.02 \pm 0.002$. Therefore most of the DM in the Universe is expected to be non-baryonic.

From a particle physics point of view, CDM is most naturally made of some weakly interacting (stable) massive particles (WIMPs). Supersymmetry (SUSY) [6], which over the years has emerged as a leading candidate for new physics beyond the Standard Model (SM), predicts that, in the presence of R-parity, the lightest supersymmetric particle (LSP) is stable. This alone makes the LSP an interesting candidate for a WIMP and DM in the Universe.

A SUSY candidate for the LSP WIMP is by no means unique. In models coupled to gravity there exists the gravitino, the SUSY partner of the graviton. The gravitino has long been known to be a potential candidate for DM, although it generically suffers from a well-known "gravitino problem" [7]. In SUSY models that incorporate the Peccei-Quinn solution to the strong CP problem, there exists the axino, the fermionic partner of the axion. The axino has recently been shown to be an attractive and well-motivated alternative candidate for the LSP and CDM [8]. A number of more speculative possibilities, stemming from, for example, string theories, have also been proposed. However, in some sense the simplest choice is to consider one of the superpartners of the SM particles as a potential LSP. Among these, the lightest neutralino $\chi$ stands out as probably the most natural and attractive candidate for the LSP and DM [9,10]. In this paper we will focus on this case. 
In order to be able to perform a reliable comparison between theoretical predictions and improving measurements of the relic abundance and limits from underground DM searches, a precise calculation of the relic density becomes necessary. The constraint (1) is known to often provide a strong restriction on allowed combinations of SUSY masses and couplings. For example, in the minimal supergravity model (also dubbed CMSSM) the condition $\Omega_{\chi} h^{2}<0.3$ provides a strong upper bound of a few hundred $\mathrm{GeV}$ on the masses of gauginos and scalars over an overwhelming fraction of the parameter space [11,12]. When combined with recent LEP and other data, the remaining CMSSM parameter space becomes highly restricted [13]. In the future, the range (1) is expected to be significantly narrowed by MAP and Planck, which in turn will lead to even stronger constraints on allowed combinations of SUSY masses and couplings.

One of the key steps in computing the relic density of the neutralino involves calculating the thermal average of its annihilation cross section times the relative velocity. This calculation is technically rather involved and, therefore, in most of the literature, approximate methods have been used. These methods are based on expanding the thermal average in powers of $x \equiv T / m_{\chi}$, where $T$ is the temperature of the thermal bath and $m_{\chi}$ is the neutralino mass. One usually computes only the first two terms of the expansion. It has been well known that the expansion fails badly near $s$-channel resonances [14 17] and near new final-state thresholds [14,15]. In particular, it was emphasized in Ref. [16] that, owing to the very narrow width of the lightest SUSY Higgs boson $h$, the error can be as large as a few orders of magnitude very close to the $h$-resonance. However, even though the usual expansion is generally expected to be accurate enough further away from resonances and thresholds, no detailed study of this point has yet been done.

On the other hand, while a general formalism for computing the thermal average precisely was derived a long time ago [15, 18], a full set of exact, general and explicit analytic expressions for the neutralino pair-annihilation cross section is still not available in the literature. (The thermal average can be obtained by performing a single integral over the cross section, as we will see below.) The cross sections for the neutralino pair-annihilation into the SM fermion-pair $(f \bar{f})$ and the lightest Higgs-boson pair $(h h)$ final states were given in Ref. [17], and for the $W W$ and $Z Z$ final states in Ref. [19]. The annihilation into $f \bar{f}$ is often most important. However, other final states can also play a considerable role, depending on the model. 
We have derived a full set of exact analytic expressions for the cross section of the neutralino pair-annihilation in the minimal supersymmetric standard model (MSSM). We have made no simplifying assumptions about the degeneracy of the left- and rightsfermion masses, and we included all tree-level final states and all intermediate states. We have kept finite widths in $s$-channel resonances. While interference terms and usually sub-dominant channels are sometimes neglected in the literature, in some cases they may give significant contributions. In particular, the channels involving gauge-Higgs boson may in some cases be sizeable or even dominant. One example where this is the case is the $W^{ \pm} H^{\mp}$ final state, and in this paper we give expressions for the cross section for the chargino exchange contribution. A full set of all the expressions for all the final states will be presented elsewhere [20].

As a check, we have performed a numerical comparison of our cross section with the results obtained by using the recently released code DarkSusy 21. We have found, for the same values of input parameters, an impressive agreement, at the level of a few per cent, for all the annihilation channels, which we find reassuring.

We have also made a comparison of the relic abundance computed using our exact formulae with the one obtained using our expansion formulae. So far, no such detailed analysis has been presented in the literature in the case of the MSSM. In Ref. [17] an analogous comparison was made in the context of the highly constrained minimal supergravity scheme and only relatively close to resonances. We confirm that the expansion gives highly inaccurate results near resonances and new thresholds. We also show that very far from such cases the error is typically rather small, of the order of a few per cent. However, we find that, because of the existence of several resonances ( $Z$ and the Higgs bosons), the expansion produces large errors, compared to an exact treatment, over a sizeable range of $m_{\chi}$, even of a several tens of $\mathrm{GeV}$. We therefore conclude that the widely used method of expansion may lead to significant errors in a sizeable fraction of the neutralino mass which is typically expected not to significantly exceed $\sim 200 \mathrm{GeV}$ by various naturalness criteria [22, 23].

In the current version of our code we have used a popular approximate iterative method of determining the freeze-out point. We have examined the accuracy of the procedure and compared our results with the ones obtained using DarkSusy. We have found that the method in general works very well when both exact and expansion methods are used, except in the latter case near resonances and thresholds where it may even break down. The iterative procedure can be safely applied in such special 
cases, provided exact expressions for the cross section are used and much care is paid to properly integrating the thermal average numerically.

\section{Calculation of the Relic Density}

The relic abundance of some stable species $\chi$ is given by $\Omega_{\chi} \equiv \rho_{\chi} / \rho_{\text {crit }}$, where $\rho_{\chi}$ is the relic's (mass) density and $\rho_{\text {crit }} \equiv 3 H_{0}^{2} / 8 \pi G=1.9 \times 10^{-29}\left(h^{2}\right) \mathrm{g} / \mathrm{cm}^{3}$ is the critical density. It can be computed by solving the Boltzmann equation, which describes the time evolution of the co-moving number density $n_{\chi}$ in the expanding Universe, ,

$$
\frac{d n_{\chi}}{d t}=-3 H n_{\chi}-\left\langle\sigma v_{\mathrm{M} \varnothing \mathrm{l}}\right\rangle\left(n_{\chi}^{2}-\left(n_{\chi}^{\mathrm{eq}}\right)^{2}\right)
$$

where $n_{\chi}^{\mathrm{eq}}$ is the number density that the species would have in thermal equilibrium, $H(T)$ is the Hubble expansion rate, $\sigma(\chi \chi \rightarrow$ all $)$ denotes the cross section of the species annihilation into ordinary particles, $v_{\mathrm{M} \varnothing \mathrm{l}}$ is a so-called Møller velocity [15 which is the relative velocity of the annihilating particles, and $\left\langle\sigma v_{\mathrm{M} \varnothing \mathrm{l}}\right\rangle$ represents the thermal average of $\sigma v_{\mathrm{M} \varnothing \mathrm{l}}$ which will be given below. In the early Universe, the species $\chi$ were initially in thermal equilibrium, $n_{\chi}=n_{\chi}^{\mathrm{eq}}$. When their typical interaction rate $\Gamma_{\chi}$ became less than the Hubble parameter, $\Gamma_{\chi} \lesssim H$, the annihilation process froze out. Since then their number in a co-moving volume has remained basically constant.

In order to calculate the relic density precisely enough, one has to consider the following ingredients: (i) compute all the contributions to the annihilation cross section, (ii) use an exact formula for the thermal average, (iii) rigorously solve the Boltzmann equation, and (iv) include co-annihilation. (This becomes important when the mass of the next LSP is nearly degenerate with the LSP mass 14,26 28].) In this work, we will concentrate on points (i) and (ii) but will also examine to some extent point (iii). In particular, we will compare the usually used expansion formulae with the exact ones. We will not consider here the effect of co-annihilation.

An often used, approximate, although in general quite accurate (see later), solution to the Boltzmann equation is based on solving iteratively the equation

$$
x_{f}^{-1}=\ln \left(\frac{m_{\chi}}{2 \pi^{3}} \sqrt{\frac{45}{2 g_{*} G_{N}}}\left\langle\sigma v_{\mathrm{M} \varnothing \mathrm{l}}\right\rangle\left(x_{f}\right) x_{f}^{1 / 2}\right),
$$

\footnotetext{
${ }^{1}$ For a review of calculations of the relic density, see, e.g., Refs. [24, 25].
} 
where $g_{*}$ represents the effective number of degrees of freedom at freeze-out $\left(\sqrt{g_{*}} \simeq 9\right)$ and $G_{N}$ is the Newton constant. Typically one finds that the freeze-out point $x_{f} \equiv$ $T_{f} / m_{\chi}$ is roughly given by $1 / 20$. The relic density $\rho_{\chi}=m_{\chi} n_{\chi}$ at present is given by

$$
\rho_{\chi}=\frac{1.66}{M_{\mathrm{Pl}}}\left(\frac{T_{\chi}}{T_{\gamma}}\right)^{3} T_{\gamma}^{3} \sqrt{g_{*}} \frac{1}{J\left(x_{f}\right)},
$$

where $M_{\mathrm{Pl}}$ denotes the Planck mass, $T_{\chi}$ and $T_{\gamma}$ are the present temperatures of the neutralino and the photon, respectively. The suppression factor $\left(T_{\chi} / T_{\gamma}\right)^{3} \approx 1 / 20$ follows from entropy conservation in a comoving volume 29. Finally, $J\left(x_{f}\right)$ is given by

$$
J\left(x_{f}\right) \equiv \int_{0}^{x_{f}} d x\left\langle\sigma v_{\mathrm{M} \varnothing \mathrm{l}}\right\rangle(x),
$$

where $x=T / m_{\chi}$, as defined earlier.

Below we will concentrate on computing $J\left(x_{f}\right)$ and in particular on the thermally averaged annihilation cross section times velocity $\left\langle\sigma v_{\mathrm{M} ø l}\right\rangle$. A proper definition which includes separate thermal baths for both annihilating particles is given by [15, 18]

$$
\left\langle\sigma v_{\mathrm{M} \varnothing \mathrm{l}}\right\rangle(T)=\frac{\int d^{3} p_{1} d^{3} p_{2} \sigma v_{\mathrm{M} \varnothing \mathrm{l}} e^{-E_{1} / T} e^{-E_{2} / T}}{\int d^{3} p_{1} d^{3} p_{2} e^{-E_{1} / T} e^{-E_{2} / T}}
$$

where $p_{1}=\left(E_{1}, \mathbf{p}_{1}\right)$ and $p_{2}=\left(E_{2}, \mathbf{p}_{2}\right)$ are the 4-momenta of the two colliding particles, and $T$ is the temperature of the bath. The above expression can be reduced to a one-dimensional integral which can be written in a Lorentz-invariant form as 15

$$
\left\langle\sigma v_{\mathrm{M} \varnothing \mathrm{l}}\right\rangle(T)=\frac{1}{8 m_{\chi}^{4} T K_{2}^{2}\left(m_{\chi} / T\right)} \int_{4 m_{\chi}^{2}}^{\infty} d s \sigma(s)\left(s-4 m_{\chi}^{2}\right) \sqrt{s} K_{1}\left(\frac{\sqrt{s}}{T}\right)
$$

where $s=\left(p_{1}+p_{2}\right)^{2}$ is a usual Mandelstam variable and $K_{i}$ denotes the modified Bessel function of order $i$.

In computing the cross section $\sigma(s)$, it is convenient to introduce a Lorentzinvariant function $w(s)$ [18

$$
w(s)=\frac{1}{4} \int d \operatorname{LIPS} \mid\left.\mathcal{A}(\chi \chi \rightarrow \text { all })\right|^{2}
$$

where $\mid\left.\mathcal{A}(\chi \chi \rightarrow$ all $)\right|^{2}$ denotes the absolute square of the reduced matrix element for the annihilation of two $\chi$ particles, averaged over initial spins and summed over final

\footnotetext{
${ }^{2}$ Note that in many cases one uses another definition of $\left\langle\sigma v_{\mathrm{M} \varnothing \mathrm{l}}\right\rangle$ which involves a single thermal bath for both neutralinos. Compare, e.g., Refs. 224, 25, 34].
} 
spins. The function $w(s)$ is related to the annihilation cross section via [17]

$$
w(s)=\frac{1}{2} \sqrt{s\left(s-4 m_{\chi}^{2}\right)} \sigma(s) .
$$

For a general annihilation process into a two-body final state $\chi \chi \rightarrow f_{1} f_{2}$, the function $w(s)$ can be written as

$$
w(s)=\frac{1}{32 \pi} \sum\left[\theta\left(s-\left(m_{f_{1}}+m_{f_{2}}\right)^{2}\right) \beta_{f}\left(s, m_{f_{1}}, m_{f_{2}}\right) \tilde{w}_{f_{1} f_{2}}(s)\right],
$$

where the summation goes over all possible channels, and

$$
\tilde{w}_{f_{1} f_{2}}(s) \equiv \frac{1}{8 \pi} \int d \Omega\left|\mathcal{A}\left(\chi \chi \rightarrow f_{1} f_{2}\right)\right|^{2},
$$

where

$$
\beta_{f}\left(s, m_{f_{1}}, m_{f_{2}}\right) \equiv\left[1-\frac{\left(m_{f_{1}}+m_{f_{2}}\right)^{2}}{s}\right]^{1 / 2}\left[1-\frac{\left(m_{f_{1}}-m_{f_{2}}\right)^{2}}{s}\right]^{1 / 2} .
$$

Computation of $w(s)$ is in general rather involved and final analytic expressions exhibit considerable complexity, as will be illustrated in the next Section. This is one reason why in most of the literature one uses the well-known approximation in terms of expansion in powers of $x,\left\langle\sigma v_{\mathrm{M} ø \mathrm{l}}\right\rangle \simeq a+b x$. Using the definition (6), the expansion reads 18

$$
<\sigma v_{\mathrm{M} \varnothing \mathrm{l}}>=\frac{1}{m_{\chi}^{2}}\left[w-\frac{3}{2}\left(2 w-w^{\prime}\right) x+\mathcal{O}\left(x^{2}\right)\right]_{s=4 m_{\chi}^{2}} \equiv a+b x+\mathcal{O}\left(x^{2}\right)
$$

where $w^{\prime}(s)$ denotes $d w(s) / d\left(s / 4 m_{\chi}^{2}\right)$.

Note that in case of the expansion of $\left\langle\sigma v_{\mathrm{M} ø \mathrm{l}}\right\rangle$ defined in terms of a single thermal bath for both neutralinos, the corresponding coefficient $a^{\prime}$ is the same as Eq. (13) while $b^{\prime}=b+\frac{3}{2} a$ [30. For more details, see, e.g., Ref. [31.

The expansion method is widely used not only because it is computationally somewhat less involved but also because it is expected to be relatively accurate. This can seen by examining the behavior of the integrand in Eq. (7). For a massive particle like the neutralino for which $T \lesssim m_{\chi} / 20$ and $\sqrt{s} \geq 2 m_{\chi}$, the argument of the function $K_{1}$ is much larger than unity. Since $K_{1}(y) \sim \sqrt{\pi / 2 y} e^{-y}$ at $y \gg 1$, the thermal average (7) can be written as a convolution of the cross section with a function $f(s)$ :

$$
\left\langle\sigma v_{\mathrm{M} ø l}\right\rangle \approx \int_{4 m_{\chi}^{2}}^{\infty} d s \sigma(s) f(s)
$$


where $f(s)$ has an exponential suppression factor, $f(s) \propto e^{-\sqrt{s} / T}$. Thus one would expect that the usual expansion in powers of $x$ should converge quickly [17].

One should however use this argument with some care. First, the annihilation cross section may change rapidly with $s$. It is well-known that this happens, e.g., near $s$-channel resonances and thresholds of new final states. In such cases the expansion method produces large errors [14 17]. Far away from such singular points the expansion is expected to give accurate results but, to our knowledge, this has never been explicitly verified in the literature. Finally, one would want to examine actually how far from resonances one can start relying on the usual approximation. As we will see later, the range of $m_{\chi}$ where the expansion fails to be reasonably accurate can be actually quite large.

\section{Analytic Results}

As stated in the Introduction, we have derived a full set of exact, analytic expressions for the cross sections for the neutralino pair-annihilation processes into all allowed (tree-level) final states in the general MSSM. We have included all contributing diagrams as well as all interference terms and kept finite widths of all $s$-channel resonances. We have made no simplifying assumptions about sfermion masses although we assumed that there are no CP violating phases in SUSY parameters. Here we will give one analytic example of our exact expressions. A full set of results for all the final states will be given elsewhere [20].

Next, starting from the exact expressions we have computed the coefficients $a$ and $b$ using Eq. (13) for all the partial annihilation channels. In the literature there exist several analytic formulae for the expansion coefficients, including [25, 32 34, but, due to different conventions and complexity of expressions, comparison is not always doable. We have checked our results for the $a$-coefficients in appropriate limits against published results and agreed in all cases. In the next Section we will present a numerical comparison of the approximation in terms of the usual expansion with our exact results.

Let us begin by introducing the relevant MSSM parameters. 3 The lightest neutralino is a mass eigenstate given by a linear combination of two neutral gauginos and

\footnotetext{
${ }^{3}$ We follow the convention of Ref. [35].
} 
two neutral higgsinos

$$
\chi=N_{11} \tilde{B}+N_{12} \tilde{W}_{3}+N_{13} \tilde{H}_{1}^{0}+N_{14} \tilde{H}_{2}^{0}
$$

The neutralino mass matrix is determined by the $U(1)_{Y}$ and $S U(2)_{L}$ gaugino mass parameters $M_{1}$ and $M_{2}$ (and we impose the usual GUT relation $M_{1}=\frac{5}{3} \tan ^{2} \theta_{W} M_{2}$ ), the Higgs/higgsino mass parameter $\mu$ and $\tan \beta=v_{2} / v_{1}$ which is the ratio of the vacuum expectation values of the two neutral Higgs fields.

There are two neutral scalar Higgs bosons $h$ and $H$ and one pseudoscalar $A$ plus a pair of charged Higgs $H^{ \pm}$. (We will typically suppress the Higgs charge assignment except where this may lead to ambiguities.) We use expressions given in Ref. [36 for computing radiatively corrected Higgs masses.

Other relevant parameters which determine the masses of scalars and various couplings are the squark soft mass parameters $m_{Q}, m_{U}$ and $m_{D}$, the slepton soft mass parameters $m_{L}$ and $m_{E}$, and the pseudoscalar mass $m_{A}$. We also include the trilinear terms $A_{i}(i=t, b, \tau)$ of the third generation which are important in determining the masses and couplings for the stop, sbottom and stau sfermions, respectively.

There are a number of final states into which the neutralino can pair-annihilate. They include: $\chi \chi \rightarrow f \bar{f}, W W, Z Z, Z h, Z H, Z A, W^{ \pm} H^{\mp}, A A, A H, A h, H H, H h$, $h h$ and $H^{+} H^{-}$. Among these, in the gaugino region, fermion pair $f \bar{f}$ final states usually give dominant contributions, unless the exchanged sfermion masses $m_{\tilde{f}}$ are large and in some special cases, as discussed earlier. These final states are also always kinematically allowed (except for $t \bar{t}$ ) but, due to the $s$-wave suppression [9], their cross section is proportional to $m_{f}^{2} / m_{W}^{2}$. For the higgsino-like neutralinos the states $Z Z$ and $W W$ become very important once kinematically allowed [19]. However, these final states can give significant contributions even in the case of the gaugino-like neutralino. This is because $W W$ and $Z Z$ are not $s$-wave suppressed, unlike the channels $h h, H h$, $H H, A A, H^{+} H^{-}$and $Z A$ [25]. What is interesting is that the cross sections for the other $s$-wave unsuppressed states $W^{ \pm} H^{\mp}, Z h, Z H, A h$ and $A H$, once kinematically allowed, can be even larger than those of $W W$ and $Z Z$. In fact, they can even dominate over those of $f \bar{f}$. These points will be illustrated with numerical examples in Section 4 .

Here, we present a set of expressions for a $t$ - and $u$-channel chargino $\left(\chi_{1,2}^{ \pm}\right)$exchange contribution to the process $\chi \chi \rightarrow W^{+} H^{-}$. This contribution is often dominant. (There are also $s$-channel diagrams involving $h, H$ and $A$ exchange. Since typically 
$m_{h} \ll m_{H} \simeq m_{A} \simeq m_{H^{ \pm}}$, the Higgs resonances will be outside of the kinematically allowed region.) The function $\tilde{w}$, Eq. (11) in this case reads

$$
\tilde{w}_{W^{+} H^{-}}^{\chi^{ \pm}}=\frac{1}{m_{W}^{2}} \sum_{i, j=1}^{2}\left[m_{\chi_{i}^{ \pm}} m_{\chi_{j}^{ \pm}} I_{i j}^{(2)}+m_{\chi} m_{\chi_{i}^{ \pm}} I_{i j}^{(1)}+I_{i j}^{(0)}\right],
$$

where

$$
\begin{aligned}
I_{i j}^{(2)}= & \left(C_{+i}^{H W^{*}} C_{+j}^{H W}+D_{+i}^{H W^{*}} D_{+j}^{H W}\right) \\
& \times\left[-\mathcal{T}_{2}-\left(s-m_{W}^{2}-m_{H^{ \pm}}^{2}-2 m_{\chi}^{2}\right) \mathcal{T}_{1}+G_{W H}^{T(1)} \mathcal{T}_{0}+6 m_{W}^{2} m_{\chi}^{2} \mathcal{Y}_{0}\right] \\
& +\left(C_{+i}^{H W^{*}} C_{+j}^{H W}-D_{+i}^{H W^{*}} D_{+j}^{H W}\right)\left[6 m_{W}^{2} m_{\chi}^{2} \mathcal{T}_{0}-\mathcal{Y}_{2}+G_{W H}^{Y(1)} \mathcal{Y}_{0}\right], \\
I_{i j}^{(1)}= & \operatorname{Re}\left(C_{+i}^{H W^{*}} C_{-j}^{H W}+D_{+i}^{H W^{*}} D_{-j}^{H W}\right)\left[-2 \mathcal{T}_{2}+2\left(2 m_{\chi}^{2}-m_{W}^{2}\right) \mathcal{T}_{1}\right. \\
& \left.-2\left(m_{\chi}^{2}-m_{W}^{2}\right)\left(m_{\chi}^{2}+2 m_{W}^{2}\right) \mathcal{T}_{0}+2 m_{W}^{2} \mathcal{Y}_{1}-G_{W H}^{Y(2)} \mathcal{Y}_{0}\right] \\
& +\operatorname{Re}\left(C_{+i}^{H W^{*}} C_{-j}^{H W}-D_{+i}^{H W^{*}} D_{-j}^{H W}\right)\left[6 m_{W}^{2} \mathcal{T}_{1}+6 m_{W}^{2}\left(m_{\chi}^{2}-m_{H}^{2}\right) \mathcal{T}_{0}\right. \\
& \left.\quad 2 \mathcal{Y}_{2}-\left(s-m_{H^{ \pm}}^{2}-4 m_{W}^{2}\right) \mathcal{Y}_{1}-G_{W H}^{Y(3)} \mathcal{Y}_{0}\right], \\
I_{i j}^{(0)}= & \left(C_{-i}^{H W^{*}} C_{-j}^{H W}+D_{-i}^{H W^{*}} D_{-j}^{H W}\right)\left[\left(s-m_{\chi}^{2}-2 m_{W}^{2}\right) \mathcal{T}_{2}-G_{W H}^{T(2)} \mathcal{T}_{1}\right. \\
& \quad\left(C_{-i}^{H W^{*}} C_{-j}^{H W}-D_{-i}^{H W^{*}} D_{-j}^{H W}\right)\left[6 m_{W}^{2} m_{\chi}^{2} \mathcal{T}_{1}-m_{\chi}^{2} \mathcal{Y}_{2}+G_{W H}^{Y(5)} \mathcal{Y}_{0}\right] . \\
& \left.\quad \mathcal{T}_{W H}+\left(s-2 m_{W}^{2}\right) \mathcal{Y}_{2}+G_{W H}^{Y(4)} \mathcal{Y}_{0}\right]
\end{aligned}
$$

Obviously the total contribution to $w(s)$ from $W^{ \pm} H^{\mp}$ is twice that from $W^{+} H^{-}$. The symbols $C_{ \pm i}^{H W}$ and $D_{ \pm i}^{H W}$ in the above equations are combinations of several coupling constants:

$$
\begin{aligned}
& C_{ \pm i}^{H W} \equiv C_{S}^{\chi_{i}^{+} \chi H^{-}} C_{V}^{\chi_{i}^{+} \chi W^{-^{*}}} \pm C_{P}^{\chi_{i}^{+} \chi H^{-}} C_{A}^{\chi_{i}^{+} \chi W^{-^{*}}} \\
& D_{ \pm i}^{H W} \equiv C_{S}^{\chi_{i}^{+} \chi H^{-}} C_{A}^{\chi_{i}^{+} \chi W^{-*}} \pm C_{P}^{\chi_{i}^{+} \chi H^{-}} C_{V}^{\chi_{i}^{+} \chi W^{-*}}
\end{aligned}
$$

The relevant couplings describe neutralino-chargino- $W^{-} / H^{-}$interaction terms in the Lagrangian as

$$
\mathcal{L}_{\mathrm{int}}=\sum_{i=1}^{2} \overline{\chi_{i}^{-}} \gamma^{\mu}\left(C_{V}^{\chi_{i}^{+} \chi W^{-}}-C_{A}^{\chi_{i}^{+} \chi W^{-}} \gamma_{5}\right) \chi W_{\mu}^{-}
$$




$$
+\sum_{i=1}^{2} \overline{\chi_{i}^{-}}\left(C_{S}^{\chi_{i}^{+} \chi H^{-}}-C_{P}^{\chi_{i}^{+} \chi H^{-}} \gamma_{5}\right) \chi H^{-}+\text {h.c. }
$$

in the convention of Ref. [35]. The two pairs of charginos are denoted by $\chi_{i}^{ \pm}(i=1,2)$ and $m_{\chi_{i}^{ \pm}}$represent their masses. The functions $\mathcal{T}_{k}=\mathcal{T}_{k}\left(s, m_{\chi}^{2}, m_{H^{ \pm}}^{2}, m_{W}^{2}, m_{\chi_{i}^{ \pm}}^{2}, m_{\chi_{j}^{ \pm}}^{2}\right)$, $\mathcal{Y}_{k}=\mathcal{Y}_{k}\left(s, m_{\chi}^{2}, m_{H^{ \pm}}^{2}, m_{W}^{2}, m_{\chi_{i}^{ \pm}}^{2}, m_{\chi_{j}^{ \pm}}^{2}\right), k=0,1,2$, and the functions $G_{W H}^{T(1-3)}, G_{W H}^{Y(1-5)}$ are listed in the Appendix.

\section{Numerical Results}

In this Section we will illustrate the points made above by presenting several numerical results. We will concentrate on the gaugino-like neutralino. We remind the reader that a nearly pure gaugino (bino) is the most natural choice for the CDM both in the MSSM [22] and in the CMSSM where in most cases the LSP comes out to be a nearly pure bino 11, 12, 16.

We begin by plotting in Figs. 1 and 2 the function $J\left(x_{f}\right)$, Eq. (5), computed using exact and expansion methods as a function of $m_{\chi}$. We take $\tan \beta=10$, $m_{0} \equiv m_{Q}=m_{U}=m_{D}=m_{L}=m_{E}=500 \mathrm{GeV}, m_{A}=400 \mathrm{GeV}, A_{t}=A_{b}=600 \mathrm{GeV}$ and $\mu=-500 \mathrm{GeV}$. To obtain sparticle and Higgs mass spectra we have used the code DarkSusy. While several analytic expressions and numerical codes (e.g., SUSPECT [37]) are available in the literature, here we have used DarkSusy to facilitate the comparison of our results for the cross sections with those produced by the package. For the values given above we obtain the following Higgs masses: $m_{h}=117.3 \mathrm{GeV}$, $m_{H}=400.5 \mathrm{GeV}$ and $m_{H^{ \pm}}=407.9 \mathrm{GeV}$.

We show both the total and the individual contributions from all the final states. The solid and dotted lines represent the exact and the expansion results, respectively. For the sake of comparison of both methods, for now we do not impose experimental constraints. Note that $m_{\chi}$ increases with increasing $M_{2}$ while we keep $\mu$ fixed at a rather large value. This means that in these Figures (and also the following ones below), the neutralino is mostly a gaugino. Even at $m_{\chi}=400 \mathrm{GeV}, N_{11}^{2}+N_{12}^{2}>0.9$. (However, between $400 \mathrm{GeV}$ and $500 \mathrm{GeV}$ the LSP turns into a nearly pure higgsino.) In this region the effect of co-annihilation with the lightest chargino and the next lightest neutralino, which we do not consider here, is not important and can be safely neglected. 
For each final state, we compute $J\left(x_{f}\right)$, Eq. (5), numerically via Eq. (17) by using our exact expressions for the cross sections $\left(J_{\text {exact }}\left(x_{f}\right)\right)$. Next, we use the cross sections to compute the first two expansion coefficients $a$ and $b$, as in Eq. (13), and to compute $J_{\exp }\left(x_{f}\right)=a x_{f}+\frac{1}{2} b x_{f}^{2}$. For consistency, in both cases we determine the freeze-out point $x_{f}$ by employing the iterative procedure (3). We will come back to the accuracy of the iterative procedure below.

In the behavior of the total $J\left(x_{f}\right)$ one can clearly recognize three peaks which are due to the $s$-channel $Z, h$ and $A / H$ exchange contributions. In particular, we can see that the $A$-resonance is very broad. (Due to a near mass degeneracy $m_{H} \simeq m_{A}$, the usually narrower $H$-pole is "burried" underneath the $A$-pole.) This is caused by the amplification of the coupling $A b \bar{b} \sim \tan \beta$ at larger $\tan \beta$ and the fact that, in the resonance region, the amplitude-square for the $A$-exchange is proportional to $s$, unlike the case of $h$ and $H$ where it goes like $s-4 m_{\chi}^{2} \approx 0$ at $\sqrt{s} \approx 2 m_{\chi}$. The $Z$-pole is also clearly visible in all the partial $f \bar{f}$ final states while the Higgs poles do not contribute to the $\nu \bar{\nu}$ final state since neutrinos do not couple to Higgs bosons. The $H$-pole also gives a visible enhancement to the $W W, Z Z$ and $h h$ final states but not to $W^{ \pm} H^{\mp}, Z A, H H, H h, A A$ and $H^{+} H^{-}$which do not become kinematically allowed until $m_{\chi}>m_{H} / 2$. Likewise, the $A$-pole shows a resonance in the $Z h$ final state but not in $W^{ \pm} H^{\mp}, Z H, A H$ and $A h$.

For the pseudoscalar Higgs exchange in $\chi \chi \rightarrow f \bar{f}$, the expansion method gives a discontinuity very close to the $A$-pole, $m_{\chi} \approx m_{A} / 2=200 \mathrm{GeV}$. For this channel, which is clearly dominant in the resonance region, the $b$-coefficient is large and negative and the expansion method and iterarative procedure break down. This can be clearly seen in all the $f \bar{f}$ windows (including $\nu \nu$ ) and also in the total value of $J_{\exp }\left(x_{f}\right)$. In the close vicinity of the poles, one can see the well-known large difference [14, 16, 17] (note a log scale) between $J_{\text {exact }}\left(x_{f}\right)$ and $J_{\exp }\left(x_{f}\right)$ while away from the poles, both contributions are similar.

We can also notice that $J_{\text {exact }}\left(x_{f}\right)$ and $J_{\text {exp }}\left(x_{f}\right)$ show a significantly different enhancement in the total values and in the up-type quark-pair final state in the region $m_{\chi} \gtrsim m_{t}=175 \mathrm{GeV}$ where the $t \bar{t}$ final state becomes kinematically allowed. In particular, $J_{\text {exact }}\left(x_{f}\right)$ increases gradually starting from $m_{\chi}$ some $10 \mathrm{GeV}$ below $m_{t}$ while $J_{\exp }\left(x_{f}\right)$ exhibits a sharp increase only at $m_{\chi}=m_{t}$. This is because the exact treatment takes into account the thermal distribution of momenta of the annihilating neutralinos, while the expansion method neglects it [14]. The same effect is visible 
near the thresholds of all the final states which only become open at some non-zero value of $m_{\chi}$, especially of those involving one or two Higgs bosons.

As we stated in Section 3, once kinematically allowed, the contributions from the $W^{ \pm} H^{\mp}$ final state can be comparable, or even larger (if sfermion masses are increased) than the $f \bar{f}$ final state. In fact, the $Z H, A h$ and, at much larger $m_{\chi}$ also $A H$, final state contributions are not much smaller. We can also see that the channels $Z Z$ and $W W$, as well as $Z h$, despite not being $s$-wave suppressed, are usually subdominant, except near $m_{\chi} \simeq m_{A} / 2$ because of the pseudoscalar Higgs exchange contributing to those channels, and when the higgsino admixture of the neutralino increases which is the case with increasing $m_{\chi}$ while keeping $\mu$ fixed.

As a way of verifying our results, we have performed a numerical comparison of our exact results for the cross section (more precisely of the function $w(s)$ ) with the same quantity computed using DarkSusy. We have found an excellent agreement at the level of a few per cent for all the tree-level final states when we fixed the same values of input parameters. This gives us some confidence that the (highly complex) expressions that we have derived (and also those in DarkSusy), are correct.

As mentioned above, in the current version of our numerical code we have used the iterative method (3) which is widely used in the literature. We applied it to both the exact formula for $\left\langle\sigma v_{\mathrm{M} ø l}\right\rangle$, Eq. (7), and to the usual approximation (13). The results are presented in Fig. 3 as a solid and dashed line, respectively. For comparison, we also display $x_{f}$ using DarkSusy (dotted line) where a different numerical procedure is used. It is clear that there is a general agreement among the three different procedures and also that $x_{f}$ can be only roughly approximated by the usually quoted value 0.05 . Away from the resonances and thresholds the expansion method can be safely used in determining $x_{f}$ while near such special points this is not the case. In particular, near the $A$-pole the expansion gives $\left\langle\sigma v_{\mathrm{M} \varnothing \mathrm{l}}\right\rangle<0$ and the iterative procedure breaks down. In contrast, the exact numerical integration (16) provides reliable results both near and away from special points. However, we have carefully verified that this is the case only when the integration in Eq. (6) is performed with high enough numerical precision.

In Fig. 4 a, we plot the ratio $\Omega_{\text {exp }} / \Omega_{\text {exact }}$ versus $m_{\chi}$ for the same set of parameters as before. We denote by $\Omega_{\text {exact }}$ and $\Omega_{\exp }$ the neutralino relic density calculated with the exact (7) and approximate (13) formulae for the thermal average, respectively. The relic abundance in both cases is computed by solving Eq. (3) iteratively and using 
Eq. (田). From now on we impose all applicable experimental constraints, including the lightest Higgs mass constraint $m_{h}>113.5 \mathrm{GeV}[38$ and a lower bound $104 \mathrm{GeV}$ for chargino masses. In the case of $b \rightarrow s \gamma$ we have followed DarkSusy in using a somewhat old range $1.0 \times 10^{-4}<B(b \rightarrow s \gamma)<4.0 \times 10^{-4}$ [39] and have not yet included new SUSY contributions at large $\tan \beta$ that have been recently pointed out [40]. We feel that this is sufficient for the purpose of this analysis. The solid line represents the experimentally allowed region while the dotted line corresponds to the low $m_{\chi}$ range excluded by collider experiments.

It is evident that the ratio $\Omega_{\exp } / \Omega_{\text {exact }}$ varies considerably with $m_{\chi}$. Near resonances it is very large as discussed above. If we conservatively demand a $10 \%$ accuracy in the relic density computations then the approximate method fails to satisfy this criterion over a broad range $160 \mathrm{GeV} \lesssim m_{\chi} \lesssim 220 \mathrm{GeV}$, which is a significant fraction of the neutralino mass range. (In fact, various naturalness constraints, even if only indicative, give a rough constraint $m_{\chi} \lesssim 200 \mathrm{GeV}$ [22,23].) For larger values of $\tan \beta$ the range of $m_{\chi}$ where $0.9<\Omega_{\exp } / \Omega_{\text {exact }}<1.1$ is not satisfied becomes even larger.

It is also clear that even further away from resonances the ratio $\Omega_{\exp } / \Omega_{\text {exact }}$ exhibits a rather complex behavior. For $220 \mathrm{GeV} \lesssim m_{\chi} \lesssim 260 \mathrm{GeV}$ the new channels $W^{ \pm} H^{\mp}, Z A$ and $Z H^{0}$ successively kick in, and become actually even somewhat more important than $f \bar{f}$. Since, as we mentioned above, the expansion method does not work properly near new channels thresholds [14], the ratio varies quite rapidly over the mentioned range of $m_{\chi}$, although not as much as near resonances. Finally, far away from resonances and thresholds, the expansion method seems to work remarkably well.

In Fig. 四 we show the relic abundance $\Omega_{\chi} h^{2}$ as a function of $m_{\chi}$. Again, the solid (dotted) line represents the range of $m_{\chi}$ allowed (excluded) by the experimental constraints. The two horizontal dashed lines delimit the cosmologically expected range (1) of $\Omega_{\chi} h^{2}$. Two features should be mentioned. Over the range of smaller $m_{\chi}$, in particular near resonances, the relic abundance varies rapidly. On the other hand, at larger $m_{\chi}$, where new channels involving the "heavy" Higgs bosons $H^{ \pm}, A$ and $H$ open up and can become important, $\Omega_{\chi} h^{2}$ exhibits a wide plateau. In both regions it is clearly important to compute $\Omega_{\chi} h^{2}$ accurately if one wants to achieve an accuracy expected from future determinations of $\Omega_{\mathrm{CDM}} h^{2}$. In particular, it is clear from Fig. 枯b that at larger $m_{\chi}$ a relatively small difference of a few per cent in determining $\Omega_{\chi} h^{2}$ could exclude on cosmological grounds the range of $m_{\chi} \gtrsim 280 \mathrm{GeV}$ which in Fig. 国b is 
(barely) allowed. This again illustrates the uncertainties involved in computing $\Omega_{\chi} h^{2}$ and in determining the cosmologically allowed regions of SUSY parameters.

\section{Conclusions}

While we are certainly still some time away from a "precision era" for measuring cosmological parameters, much effort has been focussed on improving theoretical calculations of the relic abundance of the neutralino. We have derived a full set of analytic expressions for the neutralino annihilation cross sections into all tree-level final states. In this paper, we have presented one example of such an expression for the neutralino annihilation into $W^{+} H^{-}$which can be even dominant. We have also computed the first two terms in the expansion of the exact formulae in powers of $x$ and we have compared the integral of the thermally averaged product of the cross section and velocity and the relic abundance computed both ways. We have confirmed the wellknown inaccuracy of the approximate method near reasonances and threshold and showed that, far away from such cases the expansion method works well. However, due to the presence of several resonances and thresholds in the MSSM, the expansion method may lead to sizeable errors over a relatively large range of the neutralino mass of up to several tens of $\mathrm{GeV}$. We have also demonstrated that the iterative way of computing the freeze-out point works rather well for both the exact and expansion methods of computing the thermal average but not in the latter case near resonances and thresholds.

\section{Acknowledgements}

T.N. would like to thank the CERN Theory Division for its kind hospitality.

\section{Appendix}

Here we give expressions for the auxiliary functions used in the text. First, we define

$$
\begin{aligned}
& D\left(s, x, y_{1}, y_{2}\right) \equiv x+\frac{y_{1}+y_{2}}{2}-\frac{s}{2}, \\
& F\left(s, x, y_{1}, y_{2}\right) \equiv \frac{1}{2} \sqrt{s-4 x} \sqrt{s-\left(\sqrt{y_{1}}+\sqrt{y_{2}}\right)^{2}} \sqrt{1-\frac{\left(\sqrt{y_{1}}-\sqrt{y_{2}}\right)^{2}}{s}} .
\end{aligned}
$$


If we define $D \equiv D\left(s, x, y_{1}, y_{2}\right), F \equiv F\left(s, x, y_{1}, y_{2}\right), t_{ \pm}\left(s, x, y_{1}, y_{2}\right) \equiv D \pm F$ and $\left(\mathcal{T}_{i}, \mathcal{Y}_{i}\right)$ $\equiv\left(\mathcal{T}_{i}, \mathcal{Y}_{i}\right)\left(s, x, y_{1}, y_{2}, z_{1}, z_{2}\right)(i=0,1,2)$, then

$$
\mathcal{F}\left(s, x, y_{1}, y_{2}, z\right)=\frac{1}{2 F} \ln \left|\frac{t_{+}\left(s, x, y_{1}, y_{2}\right)-z}{t_{-}\left(s, x, y_{1}, y_{2}\right)-z}\right|,
$$

and

$$
\begin{aligned}
& \mathcal{T}_{0}=\frac{1}{z_{1}-z_{2}}\left[\mathcal{F}\left(s, x, y_{1}, y_{2}, z_{1}\right)-\mathcal{F}\left(s, x, y_{1}, y_{2}, z_{2}\right)\right] \\
& \mathcal{T}_{1}=\frac{1}{z_{1}-z_{2}}\left[z_{1} \mathcal{F}\left(s, x, y_{1}, y_{2}, z_{1}\right)-z_{2} \mathcal{F}\left(s, x, y_{1}, y_{2}, z_{2}\right)\right] \\
& \mathcal{T}_{2}=1+\frac{1}{z_{1}-z_{2}}\left[z_{1}^{2} \mathcal{F}\left(s, x, y_{1}, y_{2}, z_{1}\right)-z_{2}^{2} \mathcal{F}\left(s, x, y_{1}, y_{2}, z_{2}\right)\right] \\
& \mathcal{Y}_{0}=\frac{1}{z_{1}+z_{2}-2 D}\left[\mathcal{F}\left(s, x, y_{1}, y_{2}, z_{1}\right)+\mathcal{F}\left(s, x, y_{1}, y_{2}, z_{2}\right)\right], \\
& \mathcal{Y}_{1}=\frac{1}{z_{1}+z_{2}-2 D}\left[2\left(z_{1}-D\right) \mathcal{F}\left(s, x, y_{1}, y_{2}, z_{1}\right)-2\left(z_{2}-D\right) \mathcal{F}\left(s, x, y_{1}, y_{2}, z_{2}\right)\right], \\
& \mathcal{Y}_{2}=1+\frac{1}{z_{1}+z_{2}-2 D}\left[z_{1}\left(z_{1}-2 D\right) \mathcal{F}\left(s, x, y_{1}, y_{2}, z_{1}\right)\right. \\
& \left.\quad+z_{2}\left(z_{2}-2 D\right) \mathcal{F}\left(s, x, y_{1}, y_{2}, z_{2}\right)\right] .
\end{aligned}
$$

The expressions for $G_{W H}^{T(i)}$ and $G_{W H}^{Y(j)}$, where $i=1,2,3$ and $j=1, \cdots, 5$, in Eqs. (17-19) are given by

$$
\begin{aligned}
G_{W H}^{T(1)} & \equiv s\left(m_{\chi}^{2}+2 m_{W}^{2}\right)-m_{\chi}^{4}-m_{\chi}^{2}\left(m_{H^{ \pm}}^{2}+3 m_{W}^{2}\right)-m_{W}^{2} m_{H^{ \pm}}^{2}, \\
G_{W H}^{T(2)} & \equiv s\left(m_{\chi}^{2}+2 m_{W}^{2}\right)-2 m_{\chi}^{4}+m_{\chi}^{2}\left(m_{H^{ \pm}}^{2}-m_{W}^{2}\right)-2 m_{W}^{2}\left(m_{H^{ \pm}}^{2}+m_{W}^{2}\right), \\
G_{W H}^{T(3)} & \equiv\left(m_{\chi}^{2}-m_{H^{ \pm}}^{2}\right)\left(m_{\chi}^{2}-m_{W}^{2}\right)\left(m_{\chi}^{2}+2 m_{W}^{2}\right), \\
G_{W H}^{Y(1)} & \equiv G_{W H}^{T(1)} \\
G_{W H}^{Y(2)} & \equiv 3 s m_{W}^{2}-12 m_{W}^{2} m_{\chi}^{2}+3 m_{W}^{2} m_{H^{ \pm}}^{2}-3 m_{W}^{4}, \\
G_{W H}^{Y(3)} & \equiv s^{2}-s\left(2 m_{\chi}^{2}+2 m_{H^{ \pm}}^{2}+3 m_{W}^{2}\right)+2 m_{\chi}^{4}+\left(2 m_{\chi}^{2}+m_{H^{ \pm}}^{2}\right)\left(m_{H^{ \pm}}^{2}+3 m_{W}^{2}\right)-2 m_{W}^{4}, \\
G_{W H}^{Y(4)} & \equiv s\left(m_{\chi}^{2}-2 m_{W}^{2}\right)\left(m_{\chi}^{2}-m_{H^{ \pm}}^{2}\right)+4 m_{W}^{2} m_{\chi}^{4}
\end{aligned}
$$




$$
\begin{gathered}
+m_{\chi}^{2}\left(m_{H^{ \pm}}^{4}-5 m_{W}^{2} m_{H^{ \pm}}^{2}+2 m_{W}^{4}\right)-2 m_{W}^{4} m_{H^{ \pm}}^{2} \\
G_{W H}^{Y(5)} \equiv s m_{\chi}^{2}\left(m_{\chi}^{2}-m_{W}^{2}\right)-m_{\chi}^{6}-m_{\chi}^{4}\left(m_{H^{ \pm}}^{2}+3 m_{W}^{2}\right)+m_{\chi}^{2} m_{W}^{2}\left(2 m_{H^{ \pm}}^{2}+3 m_{W}^{2}\right) .
\end{gathered}
$$

\section{References}

[1] W. Freedman, Phys. Rep. 333, 13 (2000).

[2] See, e.g., J.M. O'Meara et.al., astro-ph/0011179.

[3] P. de Bernardis et.al., Nature 404, 955 (2000).

[4] A. Balbi et.al., astro-ph/0005124.

[5] M.S. Turner and J.A. Tyson, Rev. Mod. Phys. 71, 145 (1999).

[6] For a review see, for instance, H.P. Nilles, Phys. Rep. 110, 1 (1984).

[7] H. Pagels and J.R. Primack, Phys. Rev. Lett. 48, 223 (1982); S. Weinberg, Phys. Rev. Lett. 48, 1303 (1982); J. Ellis, J.E. Kim, and D.V. Nanopoulos, PLB 145 , 181 (1984).

[8] L. Covi, J.E. Kim and L. Roszkowski, Phys. Rev. Lett. 82, 4180 (1999); L. Covi, H.B. Kim, J.E. Kim and L. Roszkowski, hep-ph/0101009.

[9] H. Goldberg, Phys. Rev. Lett. 50, 1419 (1983).

[10] J. Ellis, et al., Nucl. Phys. B 238, 453 (1984).

[11] R.G. Roberts and L. Roszkowski, Phys. Lett. B 309, 329 (1993).

[12] G.L. Kane, C. Kolda, L. Roszkowski, and J.D. Wells, Phys. Rev. D 49, 6173 (1994).

[13] For a very recent analysis, see J. Ellis, et al., hep-ph/0102098.

[14] K. Griest and D. Seckel, Phys. Rev. D 43, 3191 (1991). 
[15] P. Gondolo and G. Gelmini, Nucl. Phys. B 360, 145 (1991).

[16] P. Nath and R. Arnowitt, Phys. Rev. Lett. 69, 725 (1992).

[17] J.L. Lopez, D.V. Nanopoulos and K. Yuan, Phys. Rev. D 48, 2766 (1993).

[18] M. Srednicki, R. Watkins and K.A. Olive, Nucl. Phys. B 310, 693 (1988).

[19] K. Griest, M. Kamionkowski, and M.S. Turner, Phys. Rev. D 41, 3565 (1990).

[20] T. Nihei, L. Roszkowski and R. Ruiz de Austri, in preparation.

[21] P. Gondolo et.al., astro-ph/0012234. The code DarkSUSY is available from http://www.physto.se/ edsjo/darksusy.

[22] L. Roszkowski, Phys. Lett. B 262, 59 (1991).

[23] S. Dimopoulos and G. Giudice, Phys. Lett. B 357, 573 (1995).

[24] E.W. Kolb and M.S. Turner, The Early Universe, Addison-Wesley (1990).

[25] G. Jungman, M. Kamionkowski and K. Griest, Phys. Rep. 267, 195 (1996).

[26] S. Mizuta and M. Yamaguchi, Phys. Lett. B 298, 120 (1993).

[27] J. Edsjö and P. Gondolo, Phys. Rev. D 56, 1879 (1997).

[28] J. Ellis, T. Falk, K.A. Olive and M. Srednicki, Astropart. Phys. 13, 181 (2000).

[29] K.A. Olive, D. Schramm and G. Steigman, Nucl. Phys. B 180, 497 (1981).

[30] M. Srednicki, private communication (1994).

[31] L. Roszkowski, Phys. Rev. D 50, 4842 (1994).

[32] J. Ellis, L. Roszkowski and Z. Lalak, Phys. Lett. B 245, 545 (1990)

[33] K.A. Olive and M. Srednicki, Nucl. Phys. B 355, 208 (1991).

[34] M. Drees and M. Nojiri, Phys. Rev. D 47, 376 (1993).

[35] H.E. Haber and G.L. Kane, Phys. Rep. 117, 75 (1985); J.F. Gunion and H.E. Haber, Nucl. Phys. B 272, 1 (1986). 
[36] S. Heinemeyer, W. Hollik and G. Weiglein, Phys. Lett. B 455, 179 (1999).

[37] A. Djouadi, J.-L. Kneur and G. Moultaka, The package SUSPECT is available at http://www.lpm.univ-montp2.fr:7082/^kneur/suspect.htm].

[38] See, e.g., P. Igo-Kemenes, http://lephiggs.web.cern.ch/LEPHIGGS/talks/index.html.

[39] M.S. Alam, et al., (CLEO Collaboration), Phys. Rev. Lett. 74, 2885 (1995).

[40] G. Degrassi, P. Gambino and G.F. Giudice,JHEP 0012, 009 (2000); M. Carena, D. Garcia, U. Nierste and C.E.M. Wagner, hep-ph/0010003. 

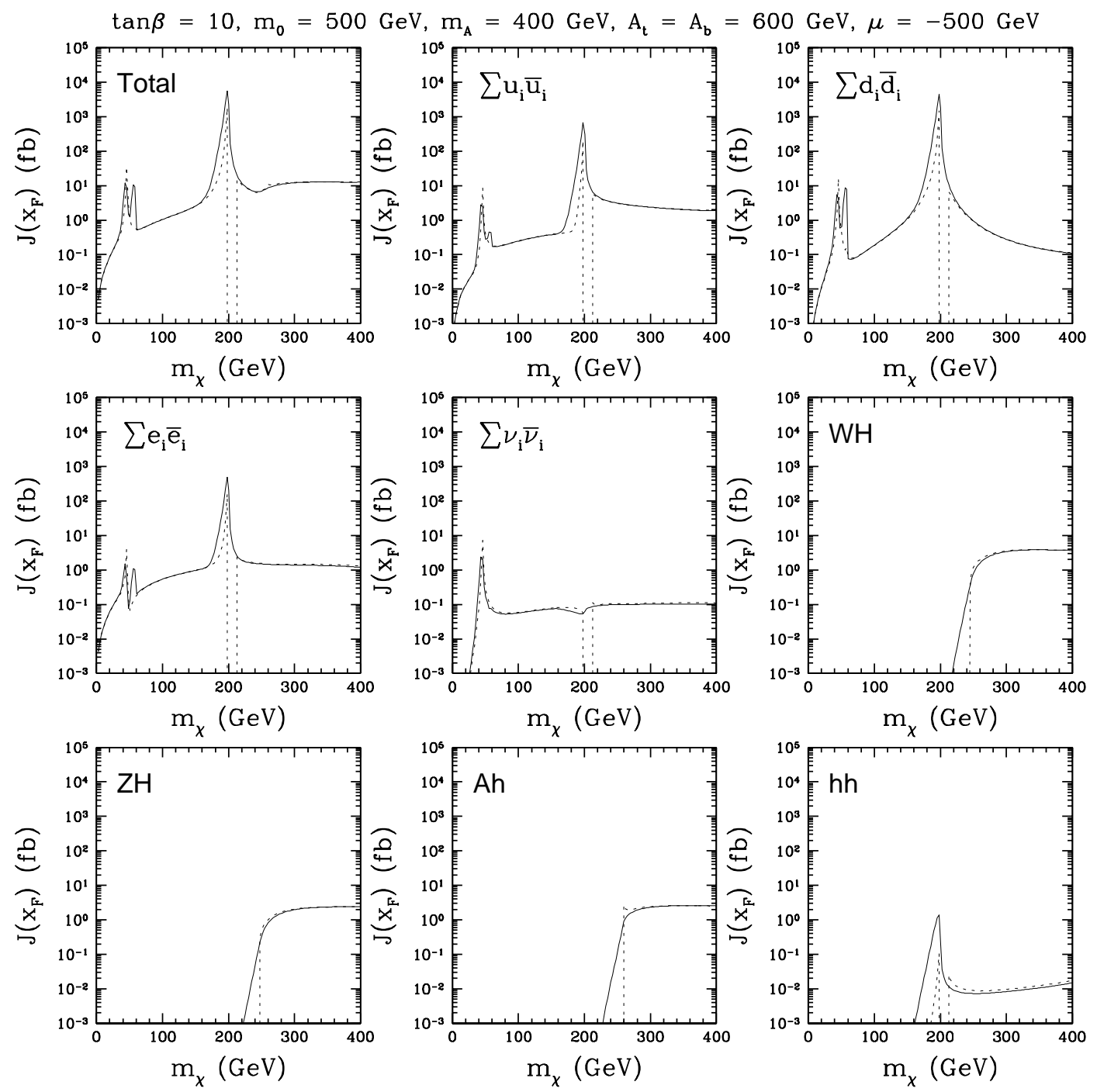

Figure 1: The total value of $J\left(x_{f}\right)$, Eq. (5), and several partial contribution are shown in separate windows as a function of $m_{\chi}$ for $\tan \beta=10, m_{0} \equiv m_{Q}=m_{U}=$ $m_{D}=m_{L}=m_{E}=500 \mathrm{GeV}, m_{A}=400 \mathrm{GeV}, A_{t}=A_{b}=600 \mathrm{GeV}$ and $\mu=-500 \mathrm{GeV}$. The solid lines represent the exact results, while the dotted ones correspond to the expansion (13). Notice that the final states $W^{ \pm} H^{\mp}, Z H^{0}$ and $A h$, once kinematically allowed, give comparable contributions to the $f \bar{f}$ channels. 

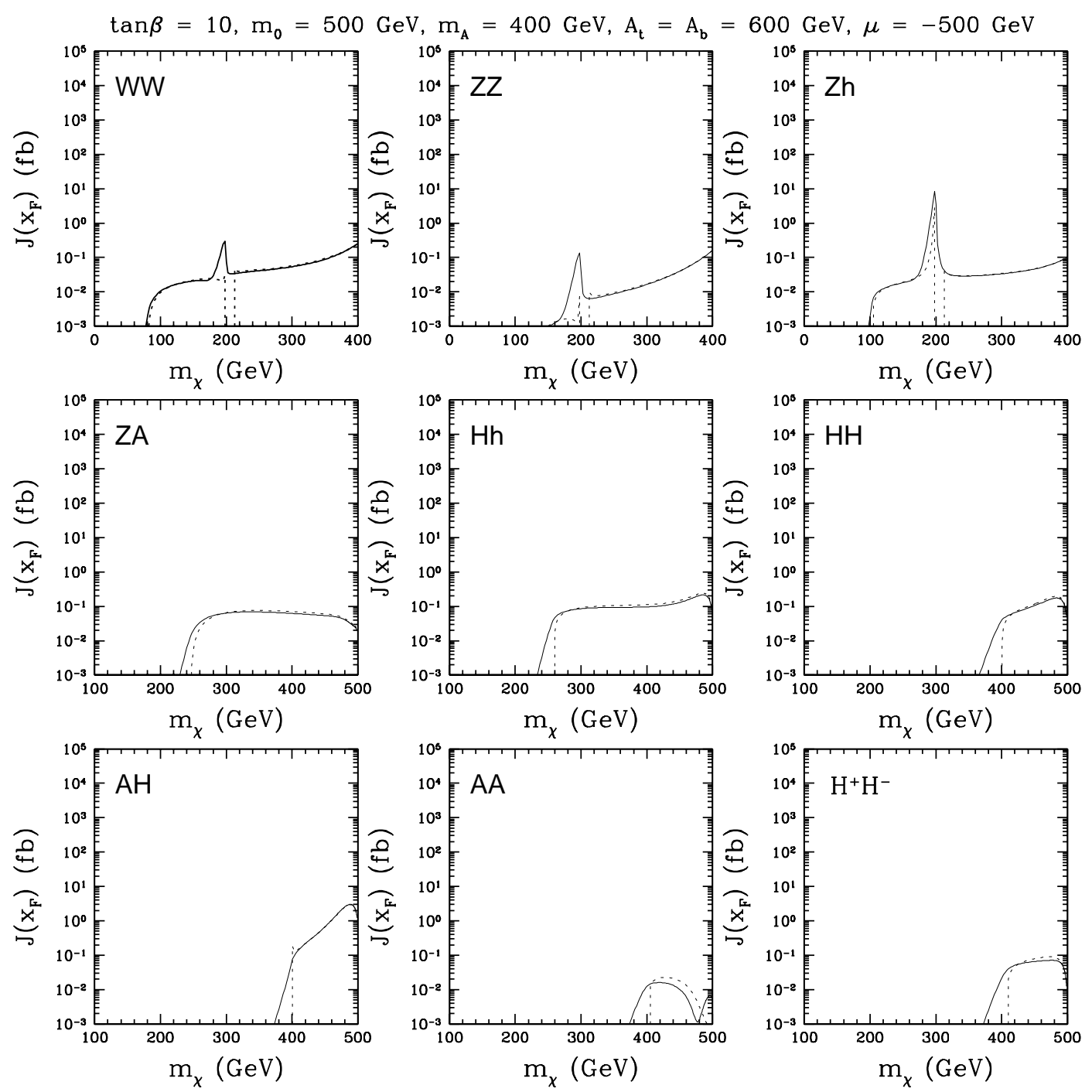

Figure 2: The same as in Fig. [1 but for mostly subdominant channels. Notice that in the lower two rows the horizontal axis has been shifted by $100 \mathrm{GeV}$. 


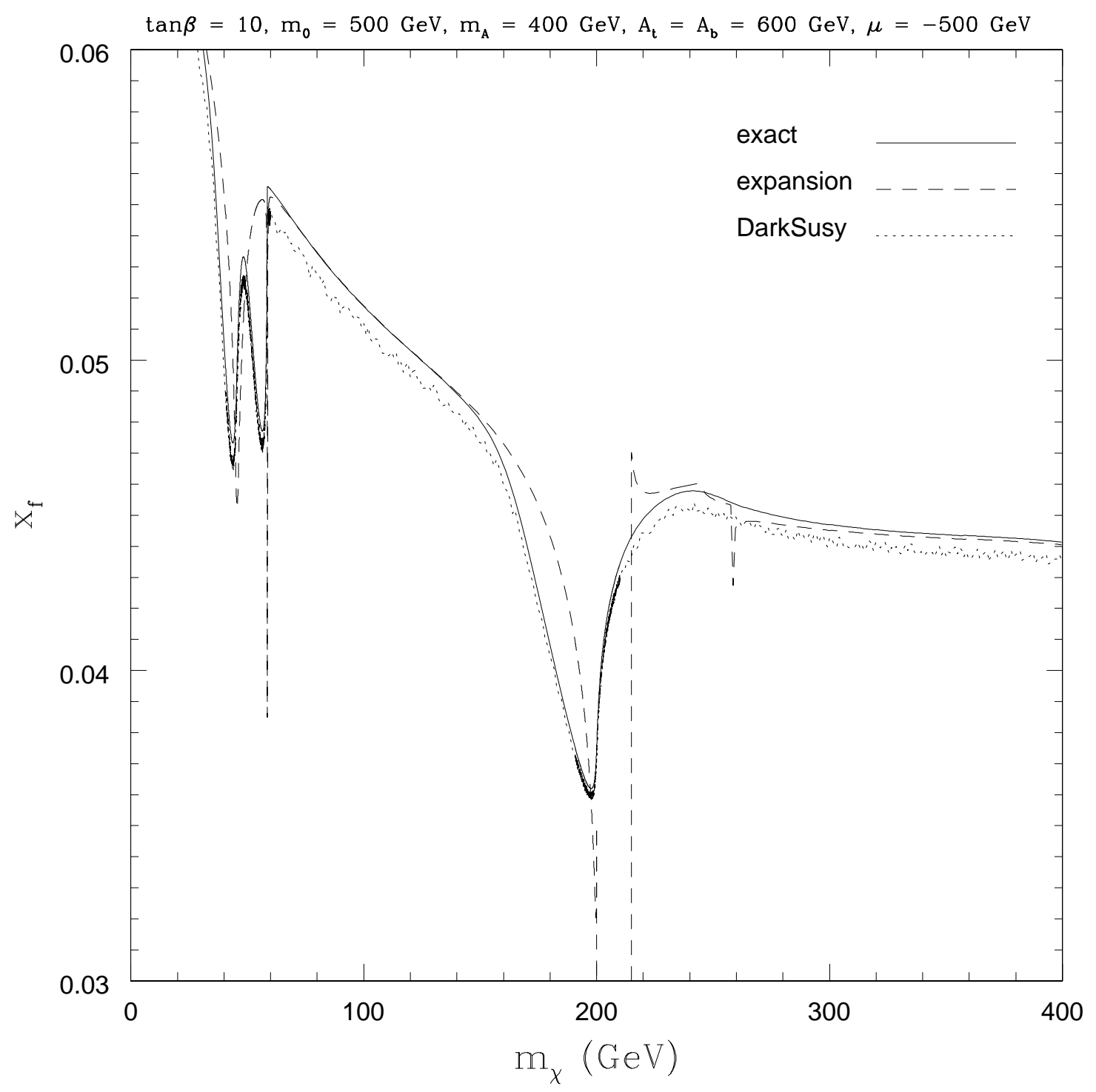

Figure 3: The freeze-out point $x_{f}$ as a function of $m_{\chi}$. The solid and dashed lines corresponds to the iterative procedure (3) with $\left\langle\sigma v_{\mathrm{M} \phi l}\right\rangle$ computed exactly (6) and in terms of the expansion (13), respectively. For comparison, the dotted line has been obtained using DarkSusy. 

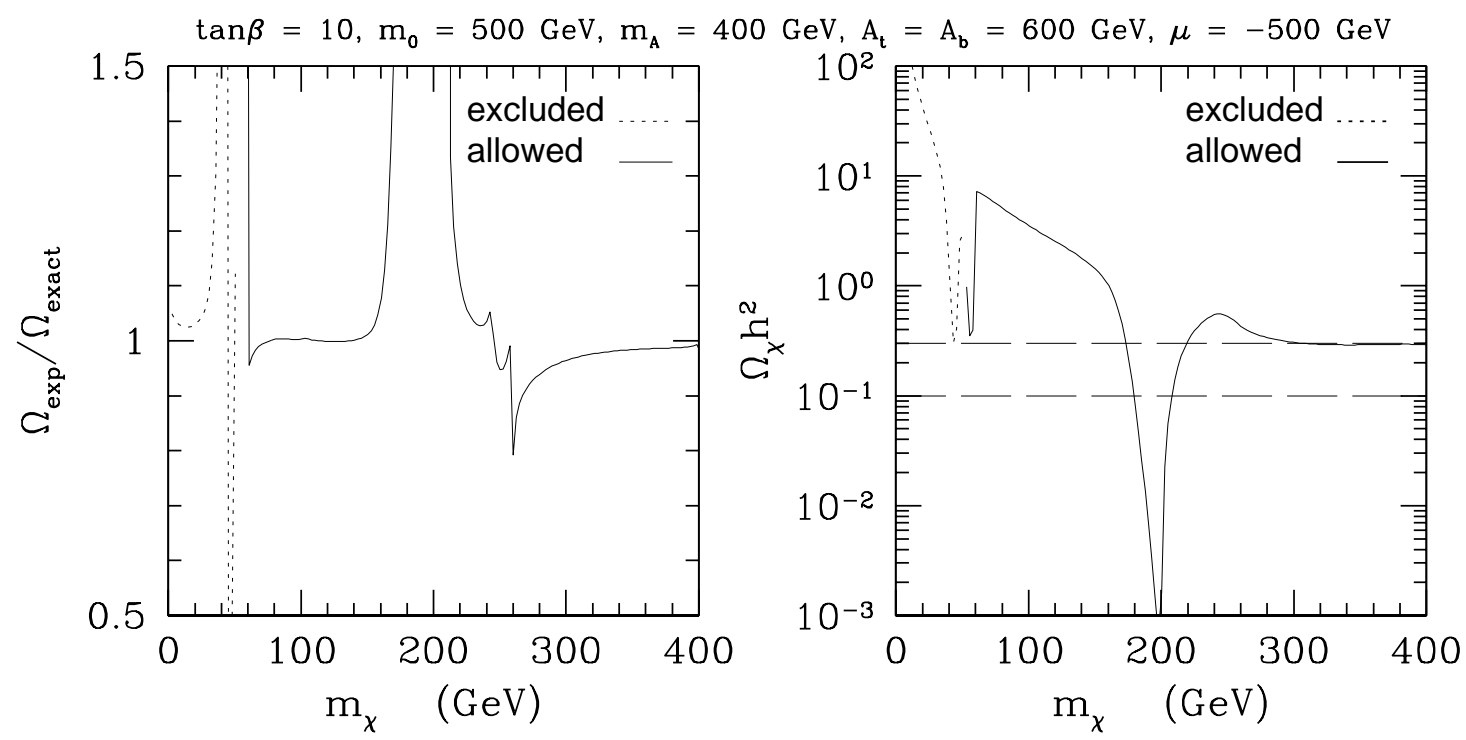

Figure 4: The ratio $\Omega_{\exp } / \Omega_{\text {exact }}$ (a) and the relic density $\Omega_{\chi} h^{2}$ (b) for the same choice of parameters as in Fig. 11. The solid (dotted) curves are allowed (excluded) by current experimental constraints. In window (a) the relic aboundance in both cases is computed by solving Eq. (3) iteratively and using Eq. (4). In window (b) we show $\Omega_{\chi} h^{2}$ is computed using our numerical code. The band between the two horizontal dashed lines corresponds to the cosmologically favoured range $0.1<\Omega_{\chi} h^{2}<0.3$. 